\title{
Surface Mail
}

National Cancer Institute

\section{Source}

National Cancer Institute. Surface Mail. NCI Thesaurus. Code C130980.

Mail that is transported by land or sea, rather than by air. 